\title{
AN INEQUALITY FOR SOME NONNORMAL OPERATORS- EXTENSION TO NORMAL APPROXIMATE EIGENVALUES
}

\author{
MASATOSHI FUJII, TAKAYUKI FURUTA, AND YUKI SEO
}

(Communicated by Palle E. T. Jorgensen)

\begin{abstract}
An inequality of use in testing convergence of eigenvector calculations is extended. It is an improvement of Bernstein and Furuta's results for selfajoint operators and dominant operators respectively.
\end{abstract}

\section{INTRODUCTION}

In [5] one of the authors showed the following theorem, which is an improvement of Bernstein's in [2].

Theorem A. If $e$ is a unit eigenvector corresponding to an eigenvalue $\lambda$ in a dominant operator $A$ on a Hilbert space $H$, then

$$
|(g, e)|^{2} \leq \frac{\|g\|^{2}\|A g\|^{2}-|(g, A g)|^{2}}{\|(A-\lambda) g\|^{2}}
$$

for all $g$ in $H$ for which $A g \neq \lambda g$.

Here an operator $A$ is called dominant if for each $\lambda$ there is a real number $M_{\lambda} \geq 1$ such that $\left\|(A-\lambda)^{*} x\right\| \leq M_{\lambda}\|(A-\lambda) x\|$ for all $x$ in $H$. We have to remark that $(A-\lambda)^{*} e=0$ under the dominance of $A$. This says that $\lambda$ is a normal eigenvalue of $A$, i.e., there is a nonzero vector $x$ in $H$ such that

$$
(A-\lambda) x=0 \text { and }(A-\lambda)^{*} x=0 .
$$

In this note, we weaken the assumption of Theorem A to dominance of the operator to normality of the eigenvalue. More precisely,

Theorem 1. If $e$ is a unit eigenvector corresponding to a normal eigenvalue $\lambda$ of $A$ on a Hilbert space $H$, then (1) holds for all $g$ in $H$ for which $A g \neq \lambda g$.

Second, we give another generalization of Theorem A to normal approximate eigenvalues [3], i.e., a complex number $\lambda$ is called a normal approximate eigenvalue of $A$ if there exists a sequence $\left\{x_{n}\right\}$ of unit vectors such that

$$
\left\|(A-\lambda) x_{n}\right\| \rightarrow 0 \text { and }\left\|(A-\lambda)^{*} x_{n}\right\| \rightarrow 0 .
$$

Received by the editors November 25, 1991.

1991 Mathematics Subject Classification. Primary 47A10; Secondary 65F15.

Key words and phrases. Eigenvector, normal eigenvalue, normal approximate eigenvalue. 
It is known in [4] that the correspondence between the normal approximate eigenvalues and the normal eigenvalues is ensured by the Berberian representations in [1]. Therefore there is a bridge from Theorem 1 to this generalization, which is of course supported by the Berberian one.

\section{RESUlts}

First of all, we cite the following lemma, which is one of the technical points in $[2,5]$.

Lemma 2. Let $A$ be an operator on a Hilbert space $H$. Then

$$
\|x\|^{2}\|A x\|^{2}-|(x, A x)|^{2}=\|x\|^{2}\|(A-\lambda) x\|^{2}-|(x,(A-\lambda) x)|^{2}
$$

for all $x$ in $H$ and complex numbers $\lambda$.

Proof of Theorem 1. Though the proof is almost the same as in [5], we give a proof for the sake of completeness. A given $g$ in $H$ is expressed as $g=\alpha e+f$, where $\alpha=(g, e)$ and $(e, f)=0$. Put $B=A-\lambda$. Then $f \neq 0, B g=B f$, so

$$
(g, B g)=(g, B f)=\alpha(e, B f)+(f, B f)=(f, B f)
$$

because $B^{*} e=0$. Hence it follows from Lemma 1 that

$$
\begin{aligned}
\frac{\|g\|^{2}\|A g\|^{2}-|(g, A g)|^{2}}{\|(A-\lambda) g\|^{2}} & =\frac{\left(|\alpha|^{2}+\|f\|^{2}\right)\|B g\|^{2}-|(g, B g)|^{2}}{\|B g\|^{2}} \\
& =|\alpha|^{2}+\frac{\|f\|^{2}\|B f\|^{2}-|(f, B f)|^{2}}{\|B f\|^{2}} \geq|\alpha|^{2} .
\end{aligned}
$$

Remark. As in the above proof, the equality holds in (1) if and only if $f$ is also an eigenvector of $A$.

Next we consider a generalization of Theorem 1 to normal approximate eigenvalues. Here we call the $\left\{e_{n}\right\}$ satisfying (3) a sequence of unit eigenvectors corresponding to a normal approximate eigenvalue $\lambda$ of $A$.

Theorem 3. If $\left\{e_{n}\right\}$ is a sequence of unit vectors corresponding to a normal approximate eigenvalue $\lambda$ of $A$, then

$$
\varlimsup\left|\left(g, e_{n}\right)\right|^{2} \leq \frac{\|g\|^{2}\|A g\|^{2}-|(g, A g)|^{2}}{\|(A-\lambda) g\|^{2}}
$$

for all $g$ in $H$ for which $A g \neq \lambda g$.

A proof of Theorem 3 will be presented in the next section.

\section{THE BERBERIAN REPRESENTATION}

We begin by recalling the Berberian representation. Following [1], we denote by Lim a fixed Banach generalized limit, defined for bounded sequences $\left\{\lambda_{n}\right\}$ of complex numbers; thus

$$
\begin{gathered}
\operatorname{Lim}\left(\lambda_{n}+\mu_{n}\right)=\operatorname{Lim} \lambda_{n}+\operatorname{Lim} \mu_{n}, \\
\operatorname{Lim}\left(\lambda \lambda_{n}\right)=\lambda \operatorname{Lim} \lambda_{n},
\end{gathered}
$$

$\operatorname{Lim} \lambda_{n}=\lim \lambda_{n}$ whenever $\left\{\lambda_{n}\right\}$ is convergent,

$\operatorname{Lim} \lambda_{n} \geq 0$ when $\lambda_{n} \geq 0$ for all $n$. 
We note that the translation invariant property of Lim is not assumed here.

Denote by $V$ the set of all bounded sequences $x=\left\{x_{n}\right\}$ with $x_{n}$ in $H$. It is clear that $V$ is a vector space relative to the definitions $x+y=\left\{x_{n}+y_{n}\right\}$ and $\lambda x=\left\{\lambda x_{n}\right\}$ for $x=\left\{x_{n}\right\}$ and $y=\left\{y_{n}\right\}$. Let $N$ be the set of all null sequences $\left\{x_{n}\right\}$ such as $\operatorname{Lim}\left(x_{n}, y_{n}\right)=0$ for all $\left\{y_{n}\right\} \in V$. Clearly $N$ is a linear subspace of $V$; we write $x^{\prime}$ for the coset $x+N$. The quotient vector space $V / N$ becomes an inner product space on defining $\left(x^{\prime}, y^{\prime}\right)=\operatorname{Lim}\left(x_{n}, y_{n}\right)$. If $x \in H$, then $\{x\}$ means the sequence of all whose terms are $x$. Since $\left(x^{\prime}, y^{\prime}\right)=(x, y)$ for $x^{\prime}=\{x\}+N$ and $y^{\prime}=\{y\}+N$, the mapping $x \rightarrow x^{\prime}$ is an isometric linear map of $H$ onto the closed subspace $H^{\prime}$ of $V / N$. Let $H^{0}$ be the completion of $V / N$. Then $H^{0}$ is an extension of $H$. For an operator $A$ acting on $H$, put

$$
A^{0}\left(\left\{x_{n}\right\}+N\right)=\left\{A x_{n}\right\}+N
$$

We can extend $A^{0}$ on $H^{0}$, which will be denoted by $A^{0}$ too. The mapping $A \rightarrow A^{0}$ of $B(H)$ into $B\left(H^{0}\right)$ is called the Berberian representation. The following theorem is proved in [1].

Theorem B (Berberian). The Berberian representation is ${ }^{*}$-isomorphic and isometric. If $A \in B(H)$, then $\pi(A)=\pi\left(A^{0}\right)=\sigma_{p}\left(A^{0}\right)$, where $\pi(A)$ is the approximate point spectrum of $A$ and $\sigma_{p}(A)$ is the point spectrum of $A$.

As a matter of fact, Theorem $B$ is used as the following form stated in the proof of [4, Theorem 1].

Theorem $\mathrm{C}$. The Berberian representation converts every normal approximate eigenvalue of $A$ into the normal eigenvalue of $A^{0}$.

Proof of Theorem 3. For each $n$, let $f_{n}$ be orthogonal vectors to $e_{n}$, and let $\alpha_{n}$ be complex numbers such that $g=\alpha_{n} e_{n}+f_{n}$. Here, let $m$ be a real vector space of bounded sequences of real numbers. Put $p(x)=\overline{\lim } x_{n}$ for $x=\left(x_{n}\right) \in m$. By the Hahn-Banach theorem, for any $x_{0} \in m$ there is a positive linear form $F$ on $m$ such that $p\left(x_{0}\right)=F\left(x_{0}\right)$ and $-p(-x) \leq F(x) \leq p(x)$ for all $x \in m$, and $F$ is clearly extended on a complex vector space of bounded sequences of complex numbers. Therefore, for a bounded sequence $\left\{\left|\alpha_{n}\right|^{2}\right\}$ there is a Banach generalized limit Lim such that $\operatorname{Lim}\left|\alpha_{n}\right|^{2}=\varlimsup\left|\alpha_{n}\right|^{2}$. Put $g^{\prime}=\{g\}, e=\left\{e_{n}\right\}$, $f=\left\{f_{n}\right\}$ in $H^{0}$, and $\alpha=\operatorname{Lim} \alpha_{n}$; then $e$ is a unit vector orthogonal to $f$ and $g^{\prime}=\alpha e+f$. Also we have

$$
|\alpha|^{2}+\|f\|^{2}=\left\|g^{\prime}\right\|^{2}=\operatorname{Lim}\left(\left|\alpha_{n}\right|^{2}+\left\|f_{n}\right\|^{2}\right)=\operatorname{Lim}\left|\alpha_{n}\right|^{2}+\|f\|^{2},
$$

that is, $\left|\left(g^{\prime}, e\right)\right|^{2}=|\alpha|^{2}=\operatorname{Lim}\left|\alpha_{n}\right|^{2}$. Furthermore, it follows from Theorem $\mathrm{C}$ that $\lambda$ is a normal eigenvalue of $A^{0}$, i.e., $A^{0} e=\lambda e$ and $A^{0 *} e=\lambda^{*} e$. Finally Theorem 1 implies that

$$
\begin{aligned}
\frac{\|g\|^{2}\|A g\|^{2}-|(g, A g)|^{2}}{\|(A-\lambda) g\|^{2}} & =\frac{\left\|g^{\prime}\right\|^{2}\left\|A^{0} g^{\prime}\right\|^{2}-\left|\left(g^{\prime}, A^{0} g^{\prime}\right)\right|^{2}}{\left\|\left(A^{0}-\lambda\right) g^{\prime}\right\|^{2}} \\
& \geq\left|\left(g^{\prime}, e\right)\right|^{2}=\operatorname{Lim}\left|\left(g, e_{n}\right)\right|^{2}=\varlimsup \lim \left|\left(g, e_{n}\right)\right|^{2} .
\end{aligned}
$$

Remark. (1) As in the proof above, put $B=A-\lambda$. Then $\left\|B^{0} f\right\|=\left\|B^{0} g^{\prime}\right\|=$ $\|B g\| \neq 0$, so $f \neq 0$. Therefore the equation holds in (4) if and only if $f$ is also an eigenvector of $B^{0}$, that is, $\left\{f_{n}\right\}$ is a sequence of approximate eigenvectors of $A$. 
(2) Finally we show a simple example of a nondominant operator, which can be covered by Theorem 3 . That is, Theorem 3 is applicable to a wider class of operators than the one of dominant operators. Actually let $M$ be the multiplication operator on $L^{2}[0,1]$, i.e., $(M f)(t)=t f(t)$ for $f \in L^{2}[0,1]$ and

$$
T=\left(\begin{array}{ll}
0 & 1 \\
0 & 0
\end{array}\right) \oplus M
$$

Then the set of all normal approximate eigenvalues of $T$ is the interval $[0,1]$ and $T$ is not dominant because the matrix is not dominant.

\section{REFERENCES}

1. S. K. Berberian, Approximate proper vectors, Proc. Amer. Math. Soc. 13 (1962), 111-114.

2. H. J. Bernstein, An inequality for selfajoint operators on a Hilbert space, Proc. Amer. Math. Soc. 100 (1987), 319-321.

3. M. Enomoto, M. Fujii, and K. Tamaki, On normal approximate spectrum, Proc. Japan Acad. 48 (1972), 211-215.

4. M. Fujii, On normal approximate spectrum. V, Proc. Japan Acad. 49 (1973), 416-419.

5. T. Furuta, An inequality for some nonnormal operators, Proc. Amer. Math. Soc. 104 (1988), 1216-1217.

Department of Mathematics, Osaka Kyoiku University, Tennoji, Osaka 543, Japan

Department of Applied Mathematics, Faculty of Science, Science University of TOKYO, SHINJUKU, TOKYo 162, JAPAN

Tennoji Senior Highschool attached to Osaka Kyoiku University, Tennoji, Osaka 543, JAPAN 